- A technique which takes very little chairside time

- Once learned this technique can be used in a flexible way for many different purposes.

- The technique maintains communication with patients when their mouths are full of

instruments.

- Enhances the patients' sense of being understood and treated sympathetically.

- This technique is a practice-builder

\title{
Visual/verbal analogue scales: Examples of brief assessment methods to aid management of child and adult patients in clinical practice
}

\author{
H. R. Chapman ${ }^{1}$ and N. Kirby-Turner ${ }^{2}$
}

The flexible use of a visual/verbal analogue scale - the thermometer - is described as a means of monitoring patients' levels of cognitive fear, trust, pain and self-esteem during treatment visits. Variations for use with very young children and those with physical and learning disabilities are also described.

Visual analogue scales are frequently used to assess and monitor self-report measures in adults and children, in particular fear and pain. The measures can be as simple as a horizontal line marked 0 and 10 or 0 and 100 at each end (Fig. 1). Sub-divisions may be marked on the line. These are used as a Likert-type scale, being marked at the appropriate point by the patient with an X. ${ }^{1}$ It is more usual to use $0-10$ for children because of developmental concepts of conservation of number. ${ }^{2}$

Another concept is that of a series of 'smiley' faces, which range from stylised 'glumness' through neutral to 'very happy'3 (Fig. 2). This type of scale has the potential problem of confusing the emotional continuum of happiness/sadness with the emotional continua of fear/courage and anxiety/confidence and the physical continuum of pain/physical comfort.

The 'fear thermometer' (Fig. 3) is a visual analogue scale frequently used to assess self-report levels of fear in children. This is often used as part of desensitisation pro-

${ }^{1 *}$ Registered Specialist in Paediatric Dentistry, 6 Woodlands Way, Southwater, Horsham, W Sussex RH13 9HZ; ${ }^{2}$ Consultant Clinical Psychologist, Department of Clinical Psychology, Mid Sussex NHS Trust, The Princess Royal Hospital, Lewes Rd, Haywards Heath, W Sussex RH16 4EX Correspondence to: Helen Chapman

E-mail:helen.chapman@tesco.net

\section{Refereed Paper}

Received 03.01.02; Accepted 03.05.02

๑ British Dental Journal 2002; 193: 447-450 grammes where children with, for example, dog phobia complete the measure before each stage in the fear hierarchy. ${ }^{4}$ The use of a thermometer gives young children a familiar practical concept to relate to. (Young children have difficulty with abstract conceptualisation. ${ }^{2}$ )

The cognitive behavioural model of dental fear has been described previously, ${ }^{5}$ stating the differences between phobia, anxiety and fearfulness in some detail. What must be reiterated is that some individuals, particularly children or adults with 'carers', may not be in a position to choose to avoid.

Our clinical methods are based on our experience of the use of such scales in treating child dental phobics in the dental surgery. The ideas were often developed in response to the specific needs of a particular individual, but were then found to be applicable to other patients, including adults.

In this article we will describe the flexible use of the 'thermometer' self-report measures during clinical situations. (For older children and adults the term 'numerical/visual analogue scale' is more appropriate.) The range of clinical information that can be gathered includes:

- Fearfulness and levels of anxiety; the monitoring of cognitive levels of fear. We will also describe various 'hands apart' versions, which are more suitable for use with the very young and with children, adolescents and adults with speech problems, learning disabilities (mental retardation), and physical disabilities.

- Monitoring of trust of the clinician

- Its use for monitoring experienced sen- 


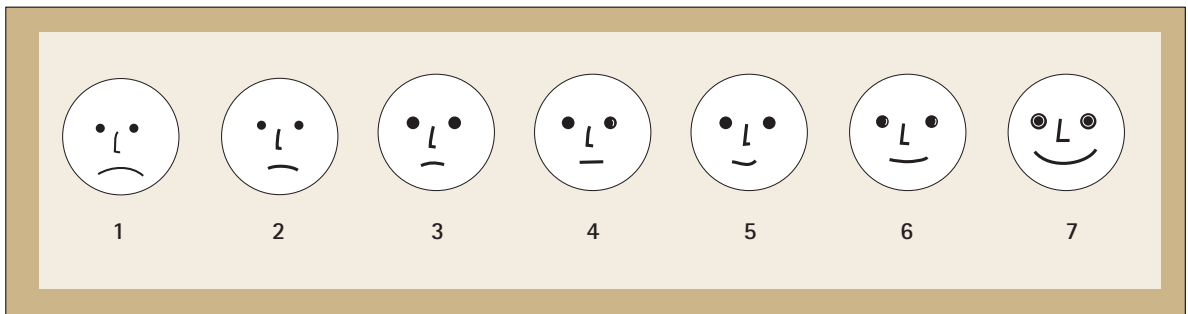

Fig. 2 'Smiley' faces series ranging from glumness to happy

sation, especially in relation to pain

- Its use as a method of monitoring and modifying perceived levels of selfesteem and coping.

Thus it can be seen that these simple measures give access to the quantity and nature of some aspects of an individual's fear. The factors currently implicated in the development of dental fear are fear of the unknown, fear of loss of control, lack of trust/fear of betrayal, fear of pain and intrusion. ${ }^{5}$

Fear is a three-axis system. It triggers:

- The physiological fight or flight mechanism of the autonomic nervous system, resulting in dilated pupils; pale, sweaty skin; trembling; pounding heart; needing to go to the toilet and feelings of nausea.

- Behavioural responses such as averting the face, fidgeting, hiding, running away or stopping procedures.

- Thoughts (cognitions) associated with the fearful situation, for example, 'I'm scared of this,' 'I can't do this,' 'I'm going to be sick'.

These three systems have been found to change, at different rates, that is they are desyncronous. ${ }^{6}$ Many dental clinicians rely on behavioural signs of fear to monitor the subjective levels of distress their patients are experiencing. Unfortunately, changes in the behavioural signs and symptoms of fear sometimes precede the cognitive changes and usually precede the physiological signs and symptoms.

Ideally, it would be useful to monitor each of these indices of fear, but there are practical difficulties with physiological monitoring. It therefore becomes important and helpful for the dentist to monitor both cognitive and behavioural levels of fear.

There are more formal pen and paper measures for the assessment of the factors influencing a person's dental fear, for example the Modified Corah Dental Anxiety Scale (MDAS), ${ }^{7}$ the Venham Picture Test $^{8}$ and the Dental Beliefs Scale. ${ }^{9}$ These are useful, particularly in retrospective audit of assessment of treatment efficacy, but cannot be used chairside to continuously monitor fear as this measure can. If reluc- tant to use a battery of pen and paper questionnaires, a careful fear history, based on the Chapman and Kirby-Turner model of dental fear, ${ }^{5}$ checking the origin of feelings, should provide all the information needed for clinical work. A record should be made on the notes of starting and final thermometer values for any specific procedure for which it is used.

We now describe the use of visual analogue scales in each of the above-mentioned domains:

\section{THE FEAR, WORRY OR ANXIETY THERMOMETER}

\section{Method of use of visual/verbal analogue} scales

A baseline level is first taken. Many research measures use scores of 0 to 8 for ease of data entry. When working clinically, we use scores of $0-10$. This is because children especially are used to being given marks out of 10 for schoolwork. Without the thermometer in front of them, some of the children find it difficult to imagine what proportion out of 8 they are experiencing. We describe ' 0 ' to the patient as, 'Feeling nice and relaxed and happy; like you do curled up on the settee at home while watching your favourite TV programme.' '10' is described as, 'The most scared/worried you can be, so scared/worried you want to run away' (ie panic). We

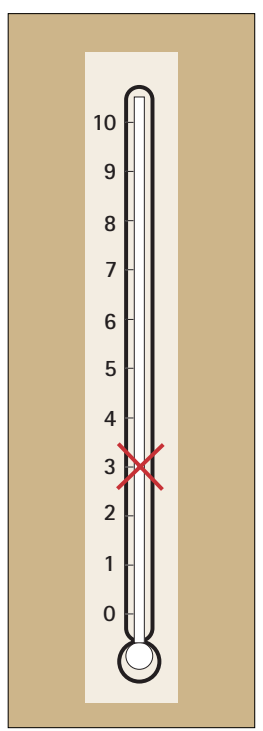

Fig. 3 The 'fear thermometer' used by children to self-report fear then ask them: 'How many out of 10 do you feel now?' Most children of six and above are capable of 'seriation' of the numbers 1 to 10 and can use this scale. ${ }^{2}$ If they cannot, then use the 'hands apart' form described below. For adult patients it is, of course, necessary to adjust the level of the language used.

This baseline figure can then be used to monitor rises and falls in experienced fearfulness during exposure, with the option of exposure to overtly non-traumatic stimuli, for example a prophylaxis brush on a finger or a sheathed needle in the mouth, being continued until the fear level drops. More threatening procedures may have to be repeated several times in quick succession until the fear level drops.

Fear levels on the thermometer can be used in conjunction with a patient hand-up stop signal. The hand is raised when the fear gets to an agreed number. This is based on the baseline number plus 1, 2 or 3 . The closer to the top of the scale, the less one can allow the fear levels to rise. When the patient's hand is raised, the procedure stops. When the patient's fear level drops back to baseline, a non-traumatic procedure is resumed. Traumatic procedures should be stopped and the patient should be carefully questioned to ascertain the cause of the problem, for example pain. Other children, often with fear levels down into single figures, may prefer to use one hand and show the number of fingers corresponding to the level of fear experienced. Another person, for example the dental nurse, may need to help monitor this if the hand is out of the dentist's immediate line of vision. If there is an element of lack of trust in the fear experience, the monitoring of the signals by the nurse can be stressed, so that the patient is confident that the bargain will be adhered to.

It is important that a stop signal is not introduced too early in the fear hierarchy. Research has shown that this actually increases the level of fear because the offering of a stop signal implies that there is something to be concerned about. ${ }^{10}$ Thus for the 'normal' patient or the moderately fearful, this should usually be offered for restorations etc, while for the terrified, particularly if they 'choke' or are intensely distrustful, it should be offered at the beginning of treatment. It is also important to warn the patient at the beginning of technique sensitive procedures such as acid etching, that once you start, you have to finish or start all over again. If you have got this far with a relaxed, confident and competent patient, there should be no problem about acceptance of this.

\section{A verbal report measure}

It is self-evident that it is relatively straight forward to ask a patient to indicate on a 
printed sheet the level of fear they are experiencing before the commencement of active treatment, but it is difficult to complete a pen and paper measure of fear when lying in a dentist's chair. A verbal report is thus extremely useful. It is important to monitor fear levels during exposure for the following reasons:

- We do not wish our patients' arousal level to rise to a point that might be beyond their coping capacity and them become panicky. The fear provoked by a simple procedure such as an examination can vary significantly within an individual patient. For example, an oral examination may be comparatively easy, except for allowing the dentist to touch a particular tooth (which has been the source of acute pain during previous treatment) with a probe. A prophylaxis may be well tolerated at the front of the mouth, but prove increasingly fear provoking as the brush/cup is moved posteriorly.

- Once the exposure has been completed, it is necessary to check that the levels of arousal have fallen to a low level before the patient is allowed to leave. Leaving with high levels of arousal is more likely to be associated with high levels of arousal on return for the next visit. This is at variance with some professional practice as dentists are very keen to reward success at task completion with immediate liberation from the surgery.

- It enhances the sense of experienced control. It thereby tends to reduce the experienced fear and hence the feelings of vulnerability because one cannot communicate with one's mouth full of dental instruments.

\section{The 'hands apart version'}

Children below the age of 6 , many adults with learning disabilities and some adults with specific language disorders may have difficulty with the concepts of conservation of number necessary for accurate use of a numerically based fear thermometer. The 'hands apart' version is a non-verbal variation that is ideal for use with these groups. It may also be very useful for very shy children who have not yet learned to trust you enough to 'open up' verbally. It is used in an identical manner to the numerical version, but the upper and lower limits are defined non-verbally. Zero is defined as hands together in front of you. The upper limit is described as arms as wide apart as you can get them. Physically demonstrate these limits and get the patient to copy you if necessary. Then ask for the current level of feeling.

Sometimes a parent or carer will report that the patient has difficulty identifying their emotions. In this case worry can be defined as 'butterflies in your tummy'. With small children you can give the tummy a small tickle to help them identify the feeling you are monitoring, but make sure you are chaperoned. You can then monitor the size of the butterflies.

Individuals, for example with hemiplegia because of cerebral palsy or a stroke, may have difficulty moving their own arms and hands. They can either rely on language or, if language or comprehension is problematic, you can demonstrate with your hands and then get the patient to move them to the correct distance apart.

For a patient with profound learning and physical disabilities, ask the carer to describe the patient's 'yes' signal. Demonstrate the hands-apart method and then ask them to use the 'yes' signal to stop you as you slowly move your hands apart.

\section{THE TRUST THERMOMETER}

Lack of trust, for some individuals, is an issue in the development of dental anxiety. It is often the direct result of intentionally or unintentionally dishonest behaviour on the part of the dentist or carer.

Patients, especially young children, may not voice these concerns directly, but will repeatedly indicate their concern by seeking reassurance. These delaying tactics can become infuriating for the busy clinician. Older children and adults may volunteer descriptions of experiences where their trust was abused. The betrayal may be hinted at in the history taking, which should be followed up with sensitive and direct questioning. Sometimes trust is satisfactory until the more challenging treatment is reached.

Limits are set to the thermometer with 0 being 'not believing/trusting at all' and 10 being 'completely believing/trusting'. Alternatively, 10 could be 'the dentist being very, very sneaky' and 0 being 'not sneaky at all.'

\section{MONITORING EXPERIENCED SENSATION - THE 'OUCH' THERMOMETER}

A visual/verbal analogue scale in the form of a numerical, 'hands apart' or 'butterfly' thermometer, which is now the 'ouch' method, can all be used directly to monitor experienced levels of pain. The lower limit is described as 'completely comfortable, not knowing it's there' and the upper limit as 'the worst pain or 'ouch' you've ever had'. Young children may need help identify/ remembering this experience with prompts from a parent/carer.

The severity of the worst physical pain will vary significantly between individuals. Broken limbs (bone pain) and serious burns are often described as the most severe pain that can be experienced. A person who has experienced, say, one of these types of pain is obviously going to have a raised upper definition of pain experience. This will influence the interpretation and tolerance of all other painlike experiences. With children, although unusual, their worst experience of pain may have been as trivial as falling over. In this case, the upper limit has to be shifted by imagining something twice or ten times as bad as that. The number of multiples will be chosen based upon the likely experience of sensation/pain to come.

Most of us have patients whose pain thresholds go through the ceiling when touched with a prophy brush. For these patients, an expansion of their pain scale is essential for them to be able to cope with the experience of dental treatment. This is because a very low pain threshold is often linked with the misinterpretation of sensations such as the vibration of the slow hand piece as pain.

You stroke the back of the patient's hand with a finger and ask them if it is painful. The answer should be 'no', so that is defined as zero. The patient is then asked to give themselves a little, gentle pinch and that is defined as 'one'. A slightly harder pinch is defined as 'two' and so on until a really vicious, nail-marking self-pinch is defined as 'ten'. The patient is then asked to go back to the trivial dental sensation and redefine it in numerical terms or, if the problem is one of misinterpretation eg vibration, to redefine it as a 'funny feeling'. If sensations are to be redefined as 'funny feelings' it is essential that there is no possibility of an element of discomfort or pain. For example, the slow handpiece should be running lightly on a restoration or the enamel of a non-pulpitic tooth.

Another common scenario is the expression of pain when the first drop of local anaesthetic takes effect. The use of topical anaesthetic, at least in the maxilla, can guarantee a pain-free passage of the needle through the mucosa. If the injection of the first drop of local anaesthetic is obviously delayed by a few seconds after the passage of the needle through the mucosa, it will be apparent whether the response is to the feelings of tingling etc of the local anaesthetic taking effect, or inadequate topical anaesthesia (which should not be a problem if anaesthesia is checked with a probe before injecting). Depending on the level of fear, trust of the dentist and cognitive ability of the patient, the technique can be described:

- As you are doing it

- Retrospectively and the patient asked to reappraise the sensation. 'Was that an ouch, or was it a 'corr'; what a funny feeling?' 
By splitting the needle entry and injection, any carer present in the surgery can make their own judgement about events, rather than rely on subsequent patient reports.

It is interesting that the belief that injections are painful is so widespread and strongly held that a child can be given a maxillary injection with no sign of pain or even mild discomfort, but when he is told what he has just had, says 'ow'. The advantage of the above technique for dentistparent relations then becomes very apparent, as it is the parent who chuckles and tells the child they are a bit late with their response.

\section{BUILDING SELF-ESTEEM AND POSITIVE STRATEGIES FOR COPING}

Visual analogue scales are also useful in developing resilience. Self-esteem is the value judgement of self against the same qualities and abilities in others. It usually develops after the age of seven. ${ }^{11}$ In the dental surgery self-esteem is based around the ability to accept treatment. Sometimes children, in learning skills necessary for accepting treatment, display a lack of faith in their own abilities; even when their newly acquired skill is pointed out to them and they are praised, their response is selfdeprecating. Be alert to this and ask how many out of ten they would give themselves for being able to do that new, specific task. If self-esteem is poor, the answer will be a value of about five. Express surprise at the low value of the figure. Ask your dental nurse what she would award out of ten. (The answer is ten.) Award ten yourself and then ask the parent/carer what they would award. The answer should be ten or above. Then return to the child and ask what they now think they should be awarded. The child's answer should have been revised upwards, hopefully to ten. Reinforce this as the correct assessment of the child's ability. Repeat the procedure at the end of subsequent tasks/sessions. Gradually, the child should reassess his ability to cope and learn in a more positive way that will lead to increased self-esteem and self-confidence.

Other methods to bolster improved self- marking include techniques such as asking, 'Who's a champ?' Prompt the reluctant to acknowledge it is him/her. This can be further reinforced by getting him to put his hands up with a positive movement (punch the air). This encourages that 'Yes!' feeling we have all experienced when we have succeeded. Also, the use of multiple senses, in this case, movement, speech and hearing, promote learning.

Ultimately, it is the sense of selfachievement that will motivate your patients, especially adolescents and adults. We should never forget that, no matter how sympathetic, kind and skilful we are as clinicians, it is our patients who have to face their fears and the ghosts of their previous experiences in order to overcome them. That takes courage.

The self-esteem thermometer should only be used at the end of a treatment section/session to boost self-esteem in those reluctant to take credit for their progress. Its use is best confined to children as adults may find this use patronising. With adults, a more direct discussion about the possibility of them not taking full credit for their achievements and how important it is to do so is more appropriate.

\section{HOW MANY THERMOMETERS AND FOR WHOM?}

This scale has multiple uses and many patients have multiple problems about accepting dental treatment. For example, a patient who has experienced pain during dental treatment and has had that pain denied is likely to fear a further painful experience, mistrust dentists and have a profound sense of loss of control. In this case, start using the thermometer early in treatment to offer control, say, for requesting rests or informing when fear levels are up to an agreed level. This will foster the patient's sense of control and foster trust. Then, when potentially painful treatment is started, the control signal, used if pain is felt, should be enough. If it becomes apparent that there is a problem with a low pain threshold/confusion with stimulation, then the thermometer can be used to modify this before treatment is resumed, backed by the use of the control thermometer.
As a general rule, only one form of the thermometer should be used at a time. Use of the Chapman and Kirby-Turner model of dental fear ${ }^{5}$ to guide dental history taking should reveal which factor is most important and thus which thermometer to use. As patients become more confident and build trust, continuous monitoring can be phased out, just leaving the patient with a simple control signal.

\section{SUMMARY}

In this article we have described the flexible use of visual/verbal analogue scales in the dental surgery. It takes very little time to describe to the patient and seconds to use as a monitoring device during treatment. If used to address the individual's problems (fear/worry, fear of pain, fear of betrayal or lack of trust, poor self-esteem and few positive coping strategies) that should have been identified during history taking, the result is a far more accurate understanding of the patient's perceptions by the dentist. In addition, the patient should feel better understood, less vulnerable and more cared for.

1. Scott PJ, Huskisson E C. Graphic representation of pain. Pain 1976: 2: 175-184.

2. Bee H. The Developing Child. 6th ed. pp249-275. New York: Harper Collins College Publishers, 1992.

3. Williams J M G, Murray J J, Lund C A, Harkiss B, de Franco A. Anxiety in the child dental clinic. J Child Psychol Psychiat 1985; 26: 305-310.

4. King N J, Hamilton D I, Ollendick T H. Children's phobias - a behavioural perspective. pp 59-71. New York: Wiley \& Sons, 1988.

5. Chapman H R, Kirby-Turner N C. Dental fear in children - a proposed model. Br Dent J 1999; 187: 408-412.

6. Hodgson R, Rachman S. Desynchrony in measures of fear. Behav Res \& Ther 1974; 12: 319-326.

7. Humphris G M, Morrison, T, Lindsay S J E. The modified dental anxiety scale: validation and United Kingdom norms. Community Dental Health 1995; 12: 143-150.

8. Venham LL, Gaulin-Kremer E. A self-report measure of situational anxiety for young children. Paediatr Dent 1979; 1: 91-96.

9. Milgrom P, Weinstein P, Getz T. Treating fearful dental patients: a patient management handbook. 2 nd ed., Revised pp 109-119. Seattle: Continuing Dental Education, 1995.

10. Corah N L. Effect of perceived control on stress reduction in pedodontic patients. J Dent Res 1973; 52: 1261-1264.

11. Bee H. The developing child. 6th ed. pp 384-389. New York: Harper Collins College Publishers, 1992. 